\title{
Experimental Results for Minimum-Time Trajectory Tracking of a Direct-Drive Three-Link Planar Arm $*$
}

\author{
Brian J. Driessen \\ Structural Dynamics Department, Sandia National Laboratories \\ Albuquerque, NM 87185-0847 \\ e-mail: bjdries@sandia.gov \\ Gordon G. Parker \\ Mechanical Engineering Dept., Michigan Institute of Technology \\ Houghton, MI 49931 \\ e-mail: ggparker@mtu.edu
}

\begin{abstract}
This work is an experimental investigation of the ability of a real three-link direct-drive arm to track model-based minimum-time trajectories that have been found offline. Sufficiently large velocity gains in the computed torque control law were not achievable with the velocity sensors described herein. This indicates the critical importance of the velocity sensing when attempting to track trajectories that push the envelope of the system's torque capabilities.
\end{abstract}

\section{Introduction}

Most experimental minimum-time trajectory tracking work for robotic arms has been for highly geared arms. (See, for example: [Kim, K., et al], [Zalzala, A., et al], [Park, J., et al], and [Dahl, O.]. This is by no means a comprehensive list.) This work, on the other hand, focuses on experimental minimum-time trajectory tracking of a real directdrive robotic arm with a $1 \mathrm{kHz}$ sampling rate, 2048-count encoders, analog tachometers, and motors driven by PWM (pulse-width-modulated) amplifiers. With such a system, to obtain a velocity measurement for feedback control, one must rely upon either the noisy tachometer signals or a numerical differentiation of the encoders. In the latter case, the velocity signal can be updated only after several sampling periods have passed (to enable division of the discrete joint-angle change by the number of sample periods passed). Such

\footnotetext{
\# Sandia is a multiprogram laboratory operated by Sandia Corporation, a Lockheed Martin Company, for the United States Department of Energy under Contract DE-AC04-94AL85000.
} 


\section{DISCLAIMER}

This report was prepared as an account of work sponsored by an agency of the United States Government. Neither the United States Government nor any agency thereof, nor any of their employees, make any warranty, express or implied, or assumes any legal liability or responsibility for the accuracy, completeness, or usefulness of any information, apparatus, product, or process disclosed, or represents that its use would not infringe privately owned rights. Reference herein to any specific commercial product, process, or service by trade name, trademark, manufacturer, or otherwise does not necessarily constitute or imply its endorsement, recommendation, or favoring by the United States Government or any agency thereof. The views and opinions of authors expressed herein do not necessarily state or reflect those of the United States Government or any agency thereof. 


\section{DISCLAIMER}

Portions of this document may be illegible in electronic image products. Images are produced from the best available original document. 
a differentiation signal is not only delayed by the number of sampling periods passed but is also only accurate to one part out of the total number of sample periods passed since the last update. (See [Driessen] for a much more detailed discussion of this issue.)

Previous known work involving direct-drive arms includes the work of [Parker, G., et al] and [Lyshevski, G.] who included linear and nonlinear, respectively, actuator electromechanical dynamics of the motors into their models to improve tracking. For the system configuration considered in the present paper, however, it is seen that even with infinite-bandwidth motors (an algebraic, not differential, relationship between motor current and motor torque), tracking of fast trajectories will still be severely limited by the lack of quality of the velocity measurements at the joints which prevents the required velocity gains in the feedback controller from being attained. In other words, even with perfect motors, much less ones with lags, the fast trajectories could not be tracked without a clean enough velocity measurement.

\section{Problem Statement}

The experimental results herein are all cases of a real three link redundant robotic arm illustrated in Figures 1 and 8. Joint angles are relative and measured counterclockwise. The arm is initially at rest, holding a position with feedback control. The dashed lines in Figures 1 and 8 represent the straight line tip paths that the arm's tip must stay on. The required terminal position of the arm's tip is the endpoint of these dashed lines. Torques are also measured counter-clockwise. Their true bounds are (starting from the hub joint): $u_{1 \max }=2.3481 \mathrm{Nm}, u_{2 \max }=0.70834 \mathrm{Nm}$, and $u_{3_{\max }}=0.29583 \mathrm{Nm}$. The joint angle bounds are: $\theta_{1 \max }=2.10 \mathrm{rad}, \theta_{2 \max }=2.28 \mathrm{rad}$, and $\theta_{3 \max }=2.13 \mathrm{rad}$. Gravity does not act in the plane of the arm. It can be shown that the inertia properties of the arm can be completely specified by the following six parameters: $A=\bar{I}_{1}+m_{1} L_{1 c}^{2}+m_{2} L_{1}^{2}+m_{3} L_{1}^{2}, B=m_{3} L_{2} L_{3 c}, C=m_{3} L_{2} L_{3 c}$, $D=\bar{I}_{3}+m_{3} L_{3 c}^{2}, E=\bar{I}_{2}+m_{2} L_{2}^{2}$, and $F=m_{2} L_{1} L_{2 c}+m_{3} L_{1} L_{2}$. where $\bar{I}_{i}$ is the centroidal rotational inertia of the ith link, $L_{i}$ the length of the ith link, and $L_{C_{i}}$ is the centroidal position of link $\mathrm{i}$, measured from the base of link $i$. The values of these parameters in the robotic arm's 
model are: $A=1.692 \mathrm{~kg} \mathrm{~m}^{2}, B=.079 \mathrm{~kg} \mathrm{~m}^{2}, C=.0829 \mathrm{~kg} \mathrm{~m}^{2}, D=.0339 \mathrm{~kg} \mathrm{~m}^{2}, E=1.199 \mathrm{~kg} \mathrm{~m}^{2}$, and $F=1.033 \mathrm{~kg} \mathrm{m^{2 }}$.

\section{Experimental Results and Discussion}

Due to inadequate sensors, the system was not able to track a minimum-time trajectory that was based upon the actual torque bounds. Instead, we had to reduce these torque bounds to about $1 / 4$ of the actual bounds in order for the arm to be able to track the associated trajectory. In the actual system, using either noisy tachometer signals that were additionally corrupted by the PWM (pulse-width-modulated) amplifiers that drive the motors or the numerically differentiated encoder signals, increasing the velocity gains beyond quite small values in the computed torque control law [see Craig] caused severe, vicious, and acoustically loud chattering of the motor torque signal between its upper and lower bounds.

Off-line simulations reveal that about $1 / 4$ max-torque trajectories were the fastest that could be tracked with the achievable velocity gains. So, in this respect, simulation and experiment matched well. Also, turning up the velocity gains in the simulations also resulted in vicious torque chattering and lack of tracking ability when simulating the bad velocity signals (either the delayed/erroneous encoder-differentiation or the noisy tach). Coloumb friction torques at the joints were experimentally estimated to be $19 \%, 11 \%$, and $7 \%$, respectively, of the torques available at the three joints. These disturbances were included in the above mentioned closed loop simulations. A $19 \%$ unmodeled disturbance torque can be expected in any real system. Such disturbances must be rejected by feedback control. Simulations indicate that with a clean enough velocity measurement and a high enough velocity gain, minimum-time trajectories based upon $70 \%$ (at least) of the difference of the maximum torque and the friction torque can be tracked and that the amount the closed loop torque will exceed the off-line (model-based) torque will be essentially the value of the friction disturbance, and nothing more. Furthermore, simulations indicated that with an $8 \mathrm{MHz}$ encoder-sampling board, the actual three-link 
arm can track such minimum-time trajectories with the same type of closed loop torque histories. (Note: such a board replaces the tachometers.) (See [Driessen] for the associated detailed set of simulations.)

The off-line trajectory for Figure 1 is shown in Figures 2 through 4 . The experimental trajectories, superimposed on top of the off-line trajectories, are shown in Figures 5 through 7. Similarly, the off-line and experimental trajectories for the problem of Figure 8 are shown in Figures 9 through 11 and 12 through 14, respectively.

We see that the joint angle tracking is quite good. However, the experimental torques are severely different from the theoretical values, differing by much more than just the friction torque. Qualitatively and non rigorously, what is happening is that without a descent velocity gain, the system cannot make "predictive" feedback corrections. This results in tiny position errors (hardly visible in Figures 5 and 12) that, when magnified by the position feedback gains in the computed torque controller, produces a significant-sized torque, thus causing the total torque to differ significantly from the theoretical torque.

\section{Conclusion}

This work presented an investigation into the ability of a real three-link planar direct-drive robotic arm to track model-based minimum-time trajectories. The investigation revealed that the real system could only track trajectories based upon about 1/4 of the actual maximum torque available at the joints. The reason was the lack of a clean enough velocity signal to allow the required velocity gains to be used, which demonstrated that a "missing link" for tracking near-full-torque minimum-time trajectories on a direct-drive arm is a clean velocity signal. Simulations indicate that an $8 \mathrm{MHz}$ encoder-sampling board would provide a velocity signal that is clean enough to allow for the required velocity gains. Future work will be to experimentally verify these simulations with the $8 \mathrm{MHz}$ encoder-sampling board. 


\section{References}

[1] Craig, J., Introduction to Robotics, Reading, Massachusetts: Addison-Wesley Publishing Company, 1987.

[2] Dahl, O., "Path Constrained Robot Control--Experimental Evaluation," Mechatronics, Vol. 4, No. 2, 1994, pp. 173-198.

[3] Driessen, B.J., PhD Dissertation, Georgia Institute of Technology, 1995.

[4] Kim, K., Kim, H., and Choi, Y., "Optimization of Cubic Polynomial Joint Trajectories and Sliding Mode Controllers for Robots Using Evolution Strategy," International Conference on Industrial Electronics, Control, and Instrumentation," Vol. 3, pp. 1444-1447.

[5] Lyshevski, S.E., "Nonlinear Dynamics and Motion Control of High-Performance Robotic Manipulators With Direct Drive Brushless Actuators," IEEE Conference on Decision and Control, Vol. 1, December 1998, pp. 937-942.

[6] Park, J., Kim, H., and Choi, Y., "Trajectory Optimization and Control for Robot Manipulator Using Evolution Strategy and Fuzzy Logic," International Conference on Systems, Man, and Cybernetics, Vol. 5, 1997, pp. 4320-4325.

[7] Parker, G.G., et al, "Optimal Trajectories for Flexible-Link Manipulator Slewing Using Recursive Quadratic Programming: Experimental Verification," Journal of Dynamic Systems, Measurement, and Control, Vol. 119, December 1997, pp. 833836.

[8] Zalzala, A. and Morris, A., "Distributed Robot Control on Transputer Network," IEEE Proceedings E, Vol. 138, No. 4, July 1991, pp. 169-176.

\section{Figures}

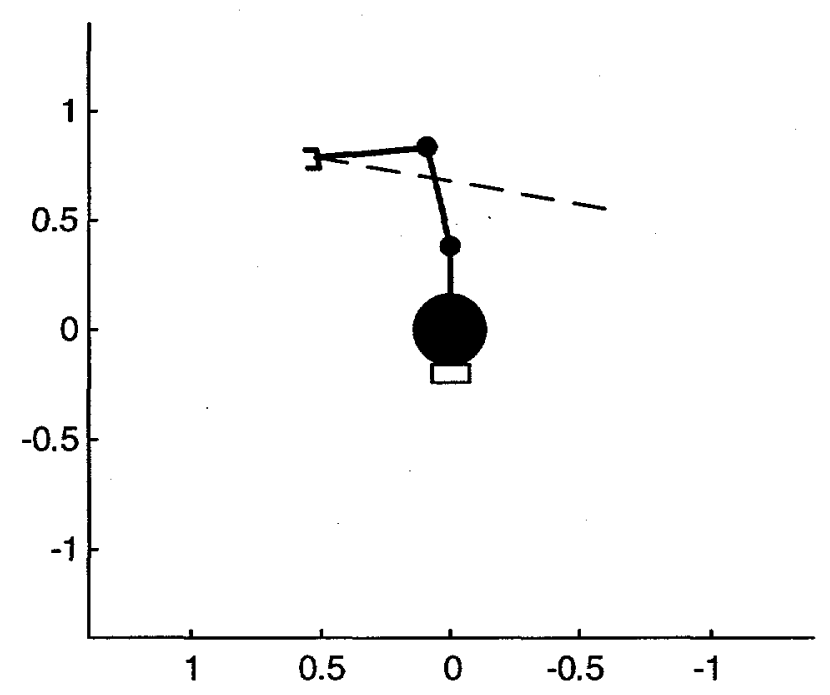

Figure 1. Case 1, Schematic of Experimental Tracking Problem 


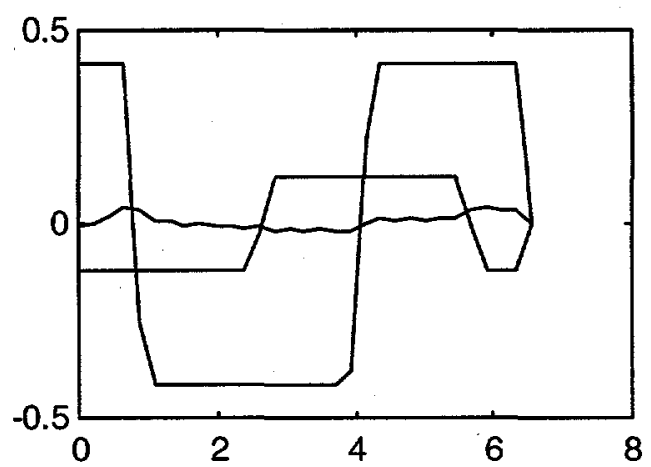

Figure 2. Case 1, Off-line Minimum-time Trajectory Torques(Nm) Versus Time (s)

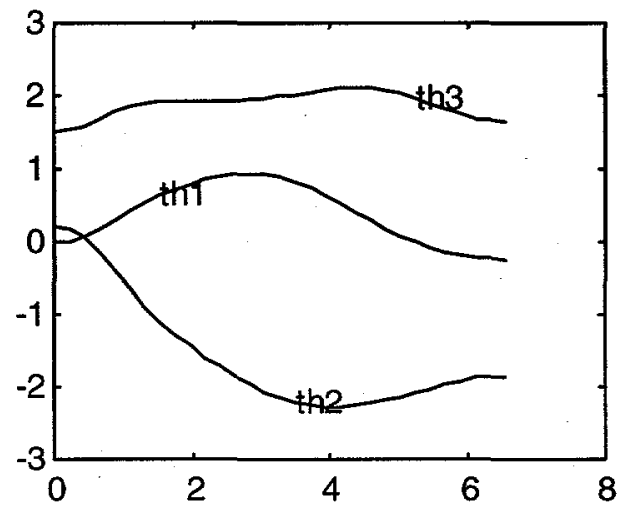

Figure 3. Case 1, Off-line Joint Angles (rad) Versus Time (s)

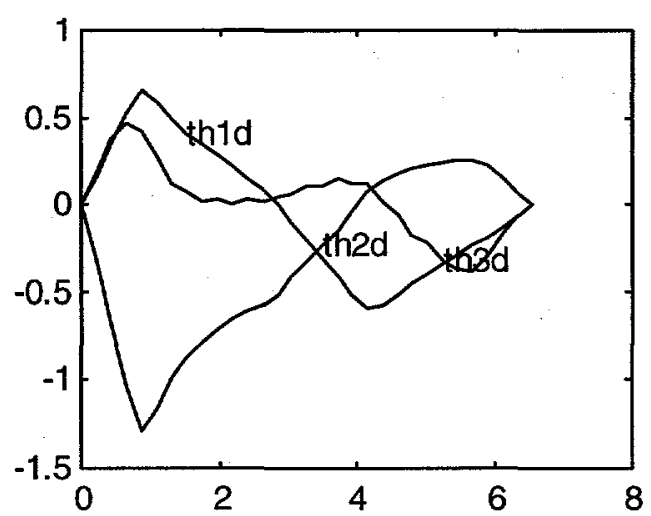

Figure 4. Case 1, Off-line Joint Velocities ( $\mathrm{rad} / \mathrm{s})$ Versus Time (s) 


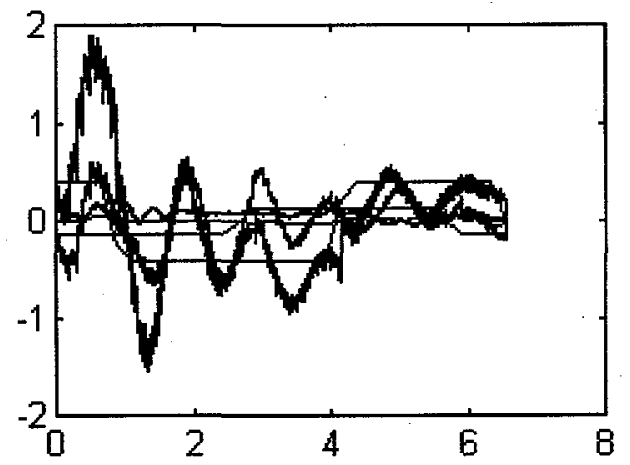

Figure 5. Case 1, Experimental and Theoretical Torques (Nm) Versus Time (s)

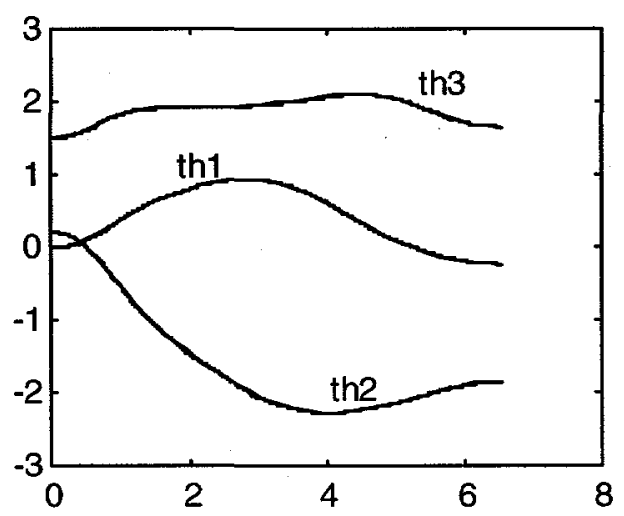

Figure 6. Case 1, Experimental and Theoretical Joint Angles (rad) Versus Time (s)

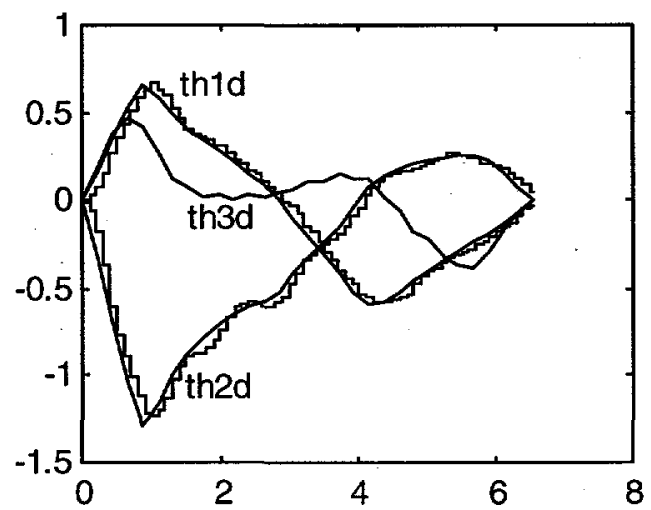

Figure 7. Case 1, Experimental and Theoretical Joint Velocities (rad/s) Versus Time (s) 


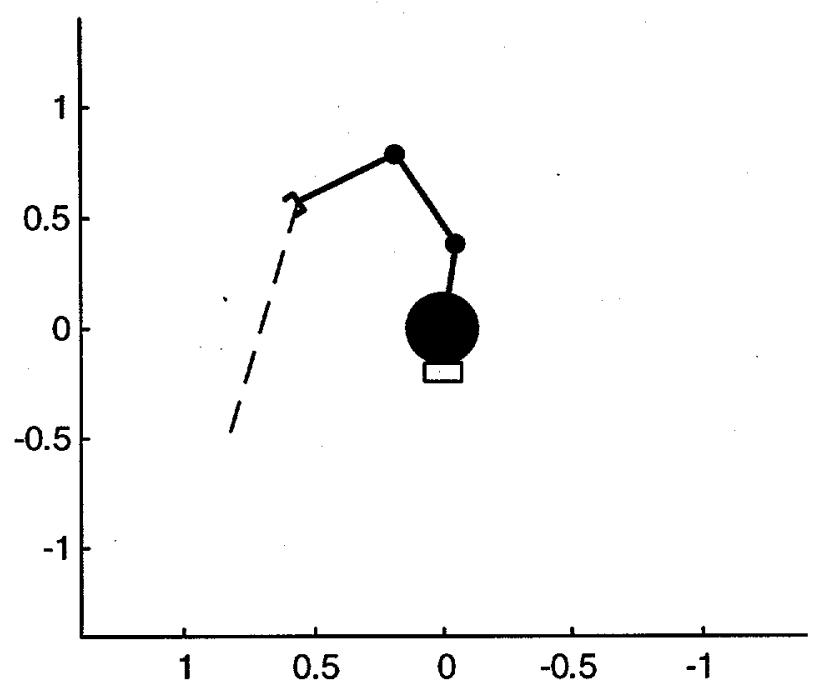

Figure 8. Case 2, Schematic of Experimental Tracking Problem

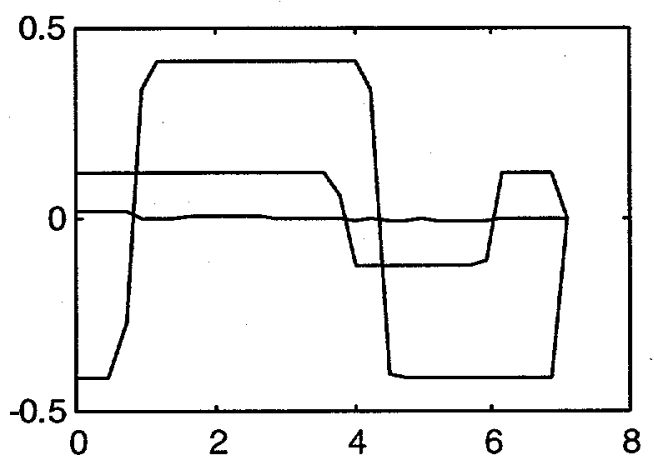

Figure 9. Case 2, Off-line Minimum-time Trajectory Torques(Nm) Versus Time (s)

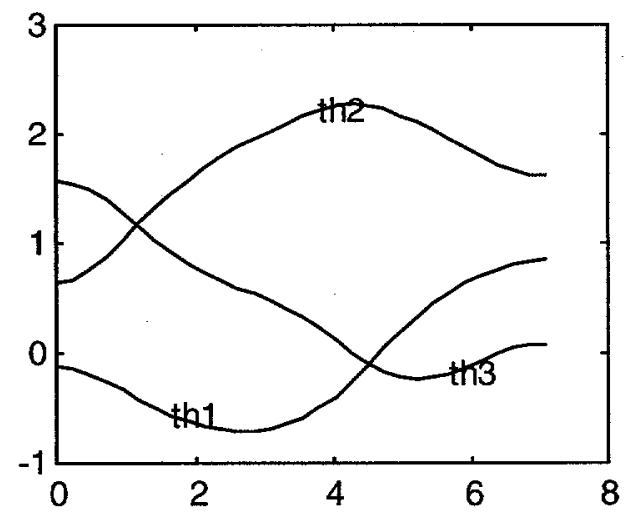

Figure 10. Case 2, Off-line Joint Angles (rad) Versus Time (s) 


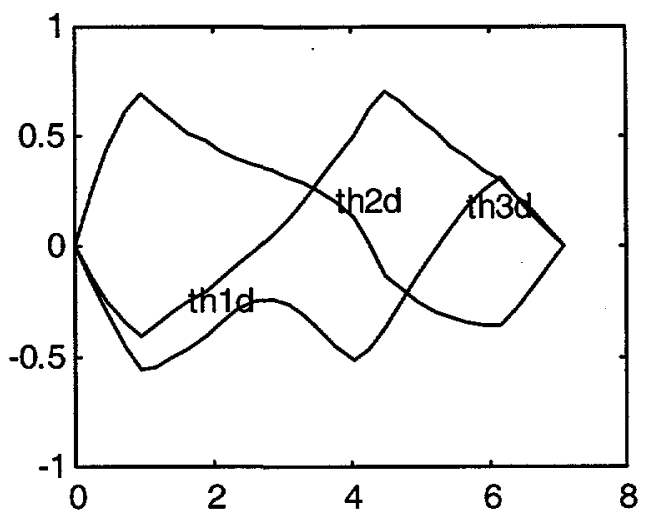

Figure 11. Case 2, Off-line Joint Velocities (rad/s) Versus Time (s)

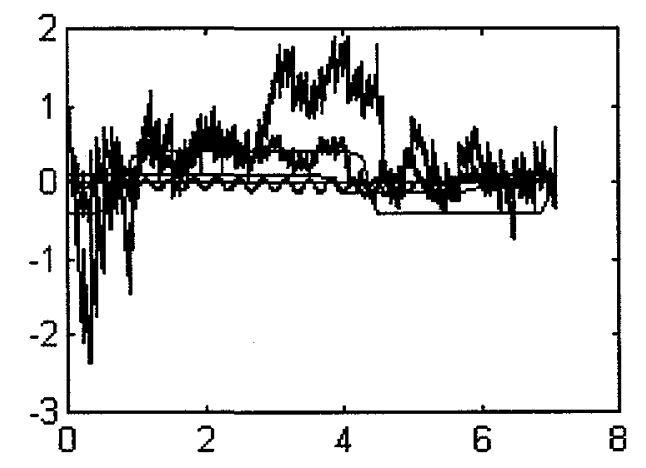

Figure 12. Case 2, Experimental and Theoretical Torques (Nm) Versus Time (s)

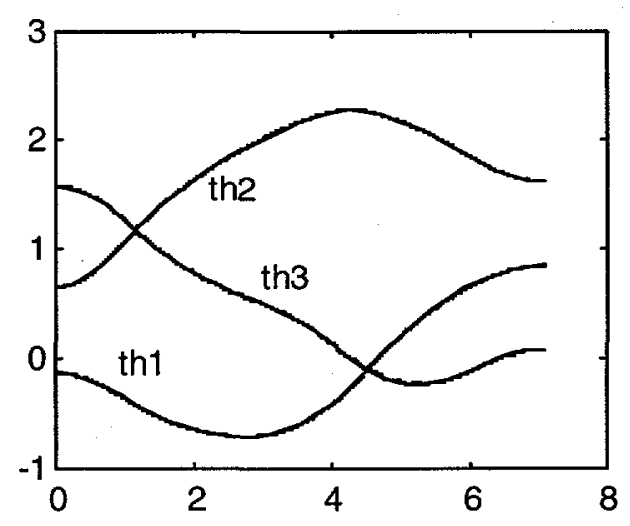

Figure 13. Case 2, Experimental and Theoretical Joint Angles (rad) Versus Time (s) 


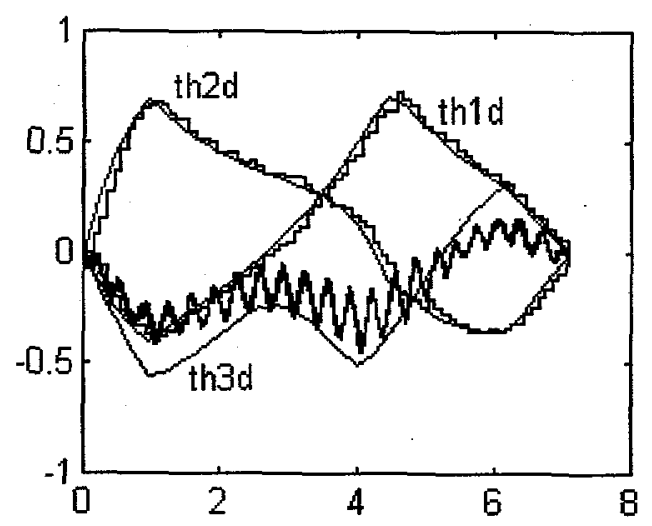

Figure 14. Case 2, Experimental and Theoretical Joint Velocities (rad/s) Versus Time (s) 